University of Nebraska - Lincoln

DigitalCommons@University of Nebraska - Lincoln

Roger Kirby Publications

Research Papers in Physics and Astronomy

May 2003

\title{
Equivalence of sweep-rate and magnetic-viscosity dynamics
}

\author{
Ralph Skomski \\ University of Nebraska-Lincoln, rskomski2@unl.edu \\ Roger D. Kirby \\ University of Nebraska-Lincoln, rkirby1@unl.edu \\ David J. Sellmyer \\ University of Nebraska-Lincoln, dsellmyer@unl.edu
}

Follow this and additional works at: https://digitalcommons.unl.edu/physics_kirby

Part of the Physics Commons

Skomski, Ralph; Kirby, Roger D.; and Sellmyer, David J., "Equivalence of sweep-rate and magnetic-viscosity dynamics" (2003). Roger Kirby Publications. 5.

https://digitalcommons.unl.edu/physics_kirby/5

This Article is brought to you for free and open access by the Research Papers in Physics and Astronomy at DigitalCommons@University of Nebraska - Lincoln. It has been accepted for inclusion in Roger Kirby Publications by an authorized administrator of DigitalCommons@University of Nebraska - Lincoln. 


\title{
Equivalence of sweep-rate and magnetic-viscosity dynamics
}

\author{
R. Skomski, ${ }^{\text {a) }}$ R. D. Kirby, and D. J. Sellmyer \\ Department of Physics and Astronomy and Center for Materials Research and Analysis, University \\ of Nebraska, Lincoln, Nebraska 68588
}

(Presented on 12 November 2002)

\begin{abstract}
The irreversible response of magnetic materials to magnetic fields of arbitrary time dependence is investigated by a master-equation approach. The magnetization reversal is expressed in terms of renormalized magnetization modes, and the resulting set of two-level master equations is solved by direct integration. The theory applies not only to linear energy-barrier laws but also to the physically more reasonable case where the activation energy is a nonlinear function of the applied field. Particular emphasis is on the relation between sweep-rate and magnetic-viscosity dynamics. Other regimes, such as oscillating magnetic fields, can be mapped onto sweep-rate dynamics. Magnetic-viscosity and sweep-rate experiments reflect the same fundamental magnetization processes, but energy barriers probed by dynamic experiments are smaller by about $20 \%$. (C) 2003 American Institute of Physics. [DOI: 10.1063/1.1557276]
\end{abstract}

\section{INTRODUCTION}

Since Ewing's 1889 discovery of the magnetic aftereffect in iron, ${ }^{1}$ the relaxation dynamics of magnetization processes has attracted much attention. ${ }^{2-8}$ For example, dynamical effects limit the thermal stability of permanent magnets, determine the long-time stability of the information stored in magnetic-recording media, and influence losses in soft magnets. Aside from a Landau-Lifshitz type damping of the spin precession, which is important at high frequencies, the relaxation reflects thermally activated jumps over energy barriers. $^{2,3}$ The corresponding relaxation time $\tau$ obeys an Arrhenius law $\tau=\tau_{0} \exp \left(E_{a} / k_{B} T\right)$, where $\tau_{0}=1 / \Gamma_{0}$ is an inverse attempt frequency of the order of $10^{-10} \mathrm{~s}$ and $E_{a}$ is the energy barrier. ${ }^{2,5,9}$ In magnetism, this mechanism is known as Néel-Brown relaxation, but the underlying physics is that of Kramers' escape-rate theory, which describes energy barriers much larger than $k_{B} T .7,10$

Depending on the experimental context, the jumps give rise to phenomena such as magnetic viscosity ${ }^{2}$ and sweeprate dependence of coercivity. ${ }^{3}$ Magnetic viscosity means that the external field $H$ is kept constant and the magnetization is monitored as a function of time, whereas sweep-rate experiments measure the hysteresis loop as a function of $\eta$ $=d H / d t .{ }^{11}$ Typical magnetic-viscosity measurements are fairly well described by the logarithmic law ${ }^{2,9,11-15}$

$$
M(H, t)=M\left(H, t_{0}\right)-S \ln \left(t / t_{0}\right) .
$$

In this equation, $\tau_{0}$ is a reference time and $S$ is the magneticviscosity constant of the magnet. Phenomenologically, $S$ $=k_{B} T \chi_{\mathrm{irr}} / M_{s} V^{*}$, where $\chi_{\mathrm{irr}}$ is the irreversible part of the susceptibility, $M_{s}$ is the spontaneous magnetization, and $V^{*}$ is known as the activation volume. ${ }^{11,16-18}$ By comparison, sweep-rate measurements yield ${ }^{3,11,19}$

\footnotetext{
${ }^{a)}$ Electronic mail: rvdskomski@msn.com
}

$$
H_{c}(\eta)=H_{c}\left(\eta_{\mathrm{ref}}\right)+\frac{k_{B} T}{M_{s} V^{*}} \ln \left(\frac{\eta}{\eta_{\mathrm{ref}}}\right)
$$

where $\eta_{\text {ref }}$ is a reference sweep rate. In Eq. (2), the dynamic contribution to the coercivity is also known as the fluctuation field. $^{20}$

Equations (1) and (2) are of great importance for the description and evaluation of the dynamics of magnetic materials. ${ }^{3,11,16-19}$ The relation between different dynamic regimes has been treated on a phenomenological level, ${ }^{21}$ but this cannot answer the question to what extent the "quasistatic" magnetic-viscosity approach $(H=$ const) is able to describe sweep-rate experiments $(d H / d t=$ const $)$. This refers, for example, to the role of nonlinear energy-barrier laws and to the question whether sweep-rate and magnetic-viscosity experiments yield the same activation volumes $V^{*}$. Note that Eqs. (1) and (2) have originally been derived from energy barriers linear in $H$, whereas energy barriers encountered in practice exhibit a nonlinear dependence on $H^{4,13,15,22}$

This work compares the magnetic-viscosity and sweeprate regimes by explicitly solving the master equation for nonlinear energy barriers. Section II casts the problem into the language of master equations, whereas Sec. III is devoted to explicit expressions for various quantities.

\section{MASTER EQUATION}

It has been known for a long time that the logarithmic law Eq. (1) can be interpreted as a superposition of elementary Barkhausen-type magnetization jumps. ${ }^{2}$ The magnetization reversal in real magnets involves multidimensional magnetic-energy landscapes $E\left(\mathbf{M}_{i}\right)$, where $\mathbf{M}_{i}=\mathbf{M}\left(\mathbf{r}_{i}\right)$ is the magnetization vector of the $i$ th atom. ${ }^{2,23,24}$ However, eigenmode analysis of the landscapes yields elementary magnetization processes having only one relevant degree of freedom $x_{\mu} \cdot{ }^{4,7,13,25}$ For example, an isolated Stoner-Wohlfarth particle is characterized by the coherent-rotation angle $x=\theta$, whereas in the case of strong domain-wall pinning $x$ describes the domain-wall position. The simultaneous involve- 
ment of several modes leads to an activation-entropy correction $^{7}$ which is of little interest in the present context. Mapping the magnetization change onto individual switching processes yields

$$
M(t, H)=-M_{s}+2 M_{s} \sum_{\mu}\left\langle x_{\mu}\right\rangle W_{\mu},
$$

where $W_{\mu}$ is the weight of each process and $\left\langle x_{\mu}\right\rangle$ denotes the thermal average of $x_{\mu}(t, H)$. Ignoring reversible contributions, we can treat $x_{\mu}$ as a binary variable; $x_{\mu}=1$ and $x_{\mu}$ $=0$ then refer to the $\uparrow$ and $\downarrow$ energy minima of the magnetization variable. Each individual process corresponds to an energy barrier $E_{\mu}$.

To describe the jumps over the energy barriers, we start from the two-state master equation ${ }^{26}$

$$
\partial P_{\mu 0} / \partial t=W_{\mu 01} P_{\mu 1}-W_{\mu 10} P_{\mu 0}
$$

and

$$
\partial P_{\mu 1} / \partial t=-W_{\mu 01} P_{\mu 1}+W_{\mu 10} P_{\mu 0} .
$$

Here $P_{\mu 0}$ and $P_{\mu 1}$ are the probabilities of having the minima $x_{\mu}=0$ and $x_{\mu}=1$ occupied, respectively, and $W_{\mu 01}$ and $W_{\mu 10}$ are the transition rates for jumping into the respective minima. The probabilities obey $P_{\mu 0}+P_{\mu 1}=1$ and $\left\langle x_{\mu}\right\rangle$ $=P_{\mu 1}$. The transition rates are $W_{\mu 10}=\Gamma_{0} \exp \left[\left(E_{\mu 0}\right.\right.$ $\left.\left.-E_{\mu}\right) / k_{B} T\right]$ and $W_{\mu 01}=\Gamma_{0} \exp \left[\left(E_{\mu 1}-E_{\mu}\right) / k_{B} T\right]$, where $E_{\mu 0}$ and $E_{\mu 1}$ are the energies of the two states and $E_{s \mu}$ is the energy of the maximum separating the $x_{\mu}=0$ and $x_{\mu}=1$ states.

\section{DYNAMICS}

Expressing $P_{\mu 0}$ and $P_{\mu 1}$ in Eq. (4) in terms of $\left\langle x_{\mu}\right\rangle$ and ignoring the small probability of jumps against the external field $\left(W_{\mu 10}=0\right)$ yields

$$
\frac{d\left\langle x_{\mu}\right\rangle}{d t}=-\Gamma_{0} \exp \left(-\frac{E_{\mu}(t)}{k_{B} T}\right)\left\langle x_{\mu}\right\rangle .
$$

The individual activation energies are given by $E_{\mu}(H)$ $=A_{\mu}\left(H_{0 \mu}-H\right)^{m_{\mu}}$, where $m_{\mu}=2$ or $m_{\mu}=3 / 2$, depending on the symmetry of the energy barriers, ${ }^{4,13,22} A_{\mu}$ is a micromagnetic parameter, and $H_{0 \mu}$ is the static switching field. The time dependence of the activation energy in Eq. (5) originates from the time dependence of the external field $H$. Equation (5) has the structure $d y / d t=a(t) y$ and solutions of the type $\ln (y)=\int a(t) d t$. Performing the integration and inserting the resulting expressions for $\left\langle x_{\mu}\right\rangle$ into Eq. (3) yields the sought-for magnetization.

In the magnetic-viscosity regime $H=$ const, the waitingtime dependence of $\left\langle x_{\mu}\right\rangle$ is given by

$$
\ln \left\langle x_{\mu}(t)\right\rangle=-f_{0} \exp \left[-A_{\mu}\left(H_{0 \mu}-H\right)^{m_{\mu}} k_{B} T\right]
$$

where $f_{0}=\Gamma_{0} t$. Switching occurs for $\left\langle x_{\mu}\right\rangle=1 / 2$, so that the dynamic switching field $H_{\mu}$ is given by the energy barrier $E_{\mu}\left(H_{\mu}\right)=k_{B} T \ln \left(\Gamma_{0} t \ln 2\right)$. This result was first obtained several decades $\mathrm{ago}^{3}$ and is, in the magnetic-recording community, also known as Sharrock's law. ${ }^{19}$ From Eq. (6) we see that the individual switching fields, including the coercivity, exhibit a logarithmic dependence on $f_{0}$ and therefore on $t$.
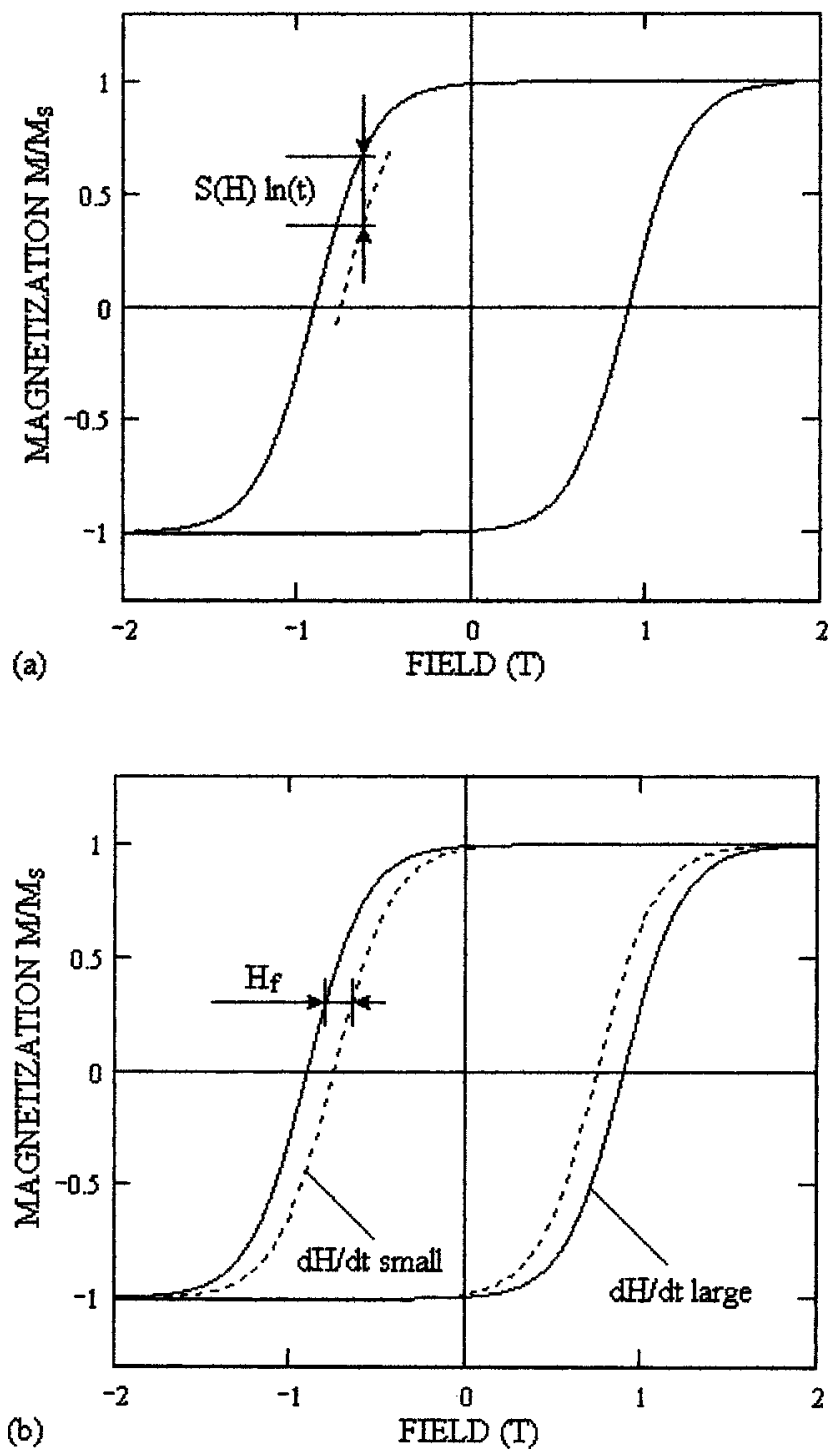

FIG. 1. Time-dependent magnetization effects: (a) magnetic viscosity and (b) sweep-rate dependence.

Using $d M(H)=\chi_{\text {irr }} d H$, where $\chi_{\text {irr }}$ is the irreversible part of the susceptibility, ${ }^{16}$ it can be shown that this translates into a logarithmic time dependence of the magnetization, as in Eq. (1). The same logarithmic dependence is obtained directly from Eq. (6), by averaging over all processes $\mu{ }^{7}$ For typical laboratory-scale measurement times of the order of $100 \mathrm{~s}$, Eq. (6) amounts to effective energy barriers $E_{\mu} \approx 25 k_{B} T$.

Sweep-rate experiments are characterized by fields $H_{z}$ $=-\eta t$, where $\eta=-d H / d t$ is the sweep rate. In this case, the solution of Eq. (5) involves a nontrivial integration. To perform the integration, we put $E_{\mu}(H)$ into Eq. (5), use the substitution $z_{\mu}(t)=\left(H_{0 \mu}-H\right)^{m} \mu$, and perform a series expansion based on $A_{\mu} \gg k_{B} T$. In lowest order, this reproduces Eq. (6) with

$$
f_{\mu}=\frac{\Gamma_{0}}{m \eta} \frac{k_{B} T}{A_{\mu}\left(H_{0 \mu}-H\right)^{m} \mu^{-1}} .
$$

Compared to the magnetic-viscosity expression $f_{0}=\Gamma_{0} t$, Eq. (7) contains a numerical factor of the order of $1 / 25$, so that the rule $E_{\mu} \approx 25 k_{B} T$ overestimates the physically encoun- 


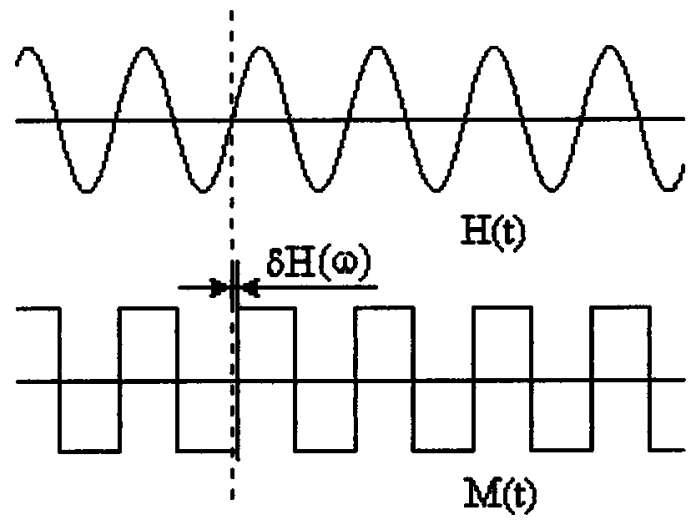

FIG. 2. High-frequency response of fine particles (schematic).

tered energy barrier. The corresponding barrier is $\mu$ dependent and somewhat difficult to predict, but taking into account that $\ln (25)=3.219$ we estimate that $E_{\mu}$ is probably closer to $20 k_{B} T$ than to $25 k_{B} T$.

\section{DISCUSSION AND CONCLUSIONS}

A physical interpretation of the comparatively small values of $f_{\mu}$ is that a sweep-rate experiment of, for example, $100 \mathrm{~s}$ per loop is much less effective in probing individual switching events than a magnetic-viscosity experiment of $100 \mathrm{~s}$. In other words, magnetic-viscosity experiments have much larger effective waiting times than sweep-rate experiments (Fig. 1). The different $f_{\mu}$ values are likely to contribute to discrepancies between sweep-rate and magneticviscosity experiments when determining, for example, activation volumes. From an experimental point of view, sweep-rate and magnetic-viscosity activation volumes are very close to each other, ${ }^{27}$ differing typically by about $20 \%$. This is compatible with our prediction, but no systematic experimental studies have been carried out to analyze the sign and magnitude of this difference and a comprehensive analysis of this point remains a challenge to future research. A related problem is the effect of the nonlinearity of the energy barriers. Linear relations such as Eqs. (1) and (2) are easily accessible by experiment but involve various linearization steps which may affect the activation volume and other magnetic parameters. ${ }^{7}$

Equation (7) is not limited to constant sweep rates but may also be used to describe any time dependence $H(t)$ so long as the time scale of the field variation remains much larger than $\tau_{0}$. The effective sweep rate is then equal to $d H(t) / d t$ determined at the switching field. Figure 2 illustrates this for a sinoidal field, $H(t)=H_{0} \sin (\omega t)$. Due to the switching character of the magnetization process, the response of individual particles is essentially rectangular, but the energy barriers lead to a delay of the response. The cor- responding loss $\mu^{\prime \prime}$ is calculated from $M(t)$ by Fourier transformation and exhibits a logarithmic frequency dependence, $\mu^{\prime \prime} \sim \ln \left(\omega / \Gamma_{0}\right)$.

In conclusion, we have used a master-equation approach to investigate the time dependence of the magnetization for different regimes $H(t)$ and for nonlinear energy barriers. The leading exponential relaxation term is the same for both the sweep-rate and magnetic-viscosity regimes, but the logarithmic factor $\ln \left(\Gamma_{0} t\right) \approx 25$ is replaced by a more complicated expression. As a rather crude estimate, the logarithmic factor for the sweep rate is about 20, that is, typical energy barriers accessed by sweep-rate experiments are smaller by about $20 \%$. The physical reason is that sweep-rate experiments are less effective in probing individual switching events than magnetic-viscosity experiments.

\section{ACKNOWLEDGMENTS}

This research is supported by AFOSR, NSF MRSEC, DOE, DARPA, and CMRA.

${ }^{1}$ J. A. Ewing, Proc. R. Soc. London 46, 269 (1889).

${ }^{2}$ R. Becker and W. Döring, Ferromagnetismus (Springer, Berlin, 1939).

${ }^{3}$ E. Kneller, in Handbuch der Physik XIII/2: Ferromagnetismus, edited by H. P. J. Wijn (Springer, Berlin, 1966), p. 438.

${ }^{4}$ R. H. Victora, Phys. Rev. Lett. 63, 457 (1989).

${ }^{5} \mathrm{~A}$. Aharoni, Introduction to the Theory of Ferromagnetism (Oxford University Press, Oxford, 1996).

${ }^{6}$ D. Givord, A. Lienard, P. Tenaud, and T. Viadieu, J. Magn. Magn. Mater. 67, L281 (1987)

${ }^{7}$ R. Skomski, R. D. Kirby, and D. J. Sellmyer, J. Appl. Phys. 85, 5069 (1999).

${ }^{8}$ H. Zeng, R. Skomski, L. Menon, Y. Liu, S. Bandyopadhyay, and D. J. Sellmyer, Phys. Rev. B 65, 134426 (2002).

${ }^{9}$ P. Gaunt, Philos. Mag. B 48, 261 (1983).

${ }^{10}$ H. A. Kramers, Physica (Amsterdam) 7, 284 (1940).

${ }^{11}$ D. J. Sellmyer, M. Yu, R. A. Thomas, Y. Liu, and R. D. Kirby, Phys. Low-Dimens. Semicond. Struct. 1-2, 155 (1998).

${ }^{12}$ R. Skomski and V. Christoph, Phys. Status Solidi B 156, K149 (1989).

${ }^{13}$ R. Skomski and J. M. D. Coey, Permanent Magnetism (Institute of Physics, Bristol, 1999).

${ }^{14}$ P. Gaunt, J. Appl. Phys. 59, 4129 (1986).

${ }^{15}$ E. Kneller, Ferromagnetismus (Springer, Berlin, 1962).

${ }^{16}$ R. Street and J. C. Wooley, Proc. Phys. Soc., London, Sect. A 62, 562 (1949)

${ }^{17}$ D. Givord, Q. Lu, M. F. Rossignol, P. Tenaud, and T. Viadieu, J. Magn. Magn. Mater. 83, 183 (1990).

${ }^{18}$ D. Givord and M. F. Rossignol, in Rare-Earth Iron Permanent Magnets, edited by J. M. D. Coey (Oxford University Press, Oxford, 1996), p. 218.

${ }^{19}$ M. P. Sharrock, J. Appl. Phys. 76, 6413 (1994).

${ }^{20}$ L. Néel, J. Phys. Radium 12, 339 (1951).

${ }^{21}$ Y. Estrin, P. G. McCormick, and R. Street, J. Phys.: Condens. Matter 1, 4845 (1989).

${ }^{22}$ R. Skomski, Thesis, Dresden 1990.

${ }^{23}$ W.-J. Chen, Sh.-F. Zhang, and H. N. Bertram, J. Appl. Phys. 71, 5579 (1992).

${ }^{24}$ A. Lyberatos and R. W. Chantrell, J. Appl. Phys. 73, 6501 (1993).

${ }^{25}$ R. Skomski and D. J. Sellmyer, J. Appl. Phys. 89, 7263 (2001).

${ }^{26}$ H. Kronmüller, Nachwirkung in Ferromagnetika (Springer, Berlin, 1968).

${ }^{27}$ D. J. Sellmyer, M. Zheng, and R. Skomski, J. Phys.: Condens. Matter 13, R433 (2001). 